\title{
Feasibility of using smart phones to estimate chlorophyll content in corn plants
}

\author{
F. VESALI', M. OMID ${ }^{*+}$, H. MOBLI*, and A. KALEITA* ${ }^{* *}$ \\ Department of Agricultural Machinery Engineering, Faculty of Agricultural Engineering and Technology, \\ University of Tehran, Karaj, Iran* \\ Department of Agricultural and Biosystems Engineering, Iowa State University, Ames, IA, USA ${ }^{* *}$
}

\begin{abstract}
New spectral absorption photometry methods are introduced to estimate chlorophyll (Chl) content of corn leaves by smart phones. The first method acquires light passing through a leaf by smartphone camera, compensating for differences in illumination conditions. In order to improve performance of the method, spectral absorption photometry (SAP) with background illumination has been considered as well. Data were acquired by smartphone camera in Iowa State University maize fields. Various indices were extracted and their correlation with Chl content were examined by Minolta SPAD-502. Hue index in SAP reached $R^{2}$ value of 0.59 . However, with light-aided SAP (LASAP), $R^{2}$ of 0.97 was obtained. Among traits, the vegetation index gave the most accurate indication. We can conclude that the high performance of LASAP method for estimating Chl content, leads to new opportunities offered by smart phones at much lower cost. This is a highly accurate alternative to SPAD meters for estimating Chl content nondestructively.
\end{abstract}

Additional key words: Android phone; color index; SPAD meter; spectral absorption photometry.

\section{Introduction}

$\mathrm{Chl}$ is the most important pigment of plants since it plays a crucial role in photosynthesis (Muñoz Huerta et al. 2013). The determination of Chl contents enables to obtain important information on plant growth and photosynthesis. Nitrogen is also one of the important elements in Chl molecules. Therefore, the $\mathrm{Chl}$ content is also an indicator of nitrogen status in plant tissues (Evans 1989). Nitrogen is a nutrient which is most required by plants. However, an excess of nitrogenous compounds, particularly in agricultural environment, leads to water pollution (Turner and Rabalais 1991) and upon intake by human bodies, it is transformed into nitrosoamine molecules acting as carcinogens.

In order to determine $\mathrm{Chl}$ contents, a Minolta $S P A D$ 502 (Japan) is one of the most used Chl measuring instruments and has been used widely by researchers to determine also a nitrogen status in different plant species (Miao et al. 2009, Vesali et al. 2015, Yadav et al. 2010).
The SPAD instrument emits a light beam of two wavelengths (940 and $650 \mathrm{~nm}$ ), from upper surface of the leaf and a device is located in exactly the same position at the bottom surface of the leaf, capturing transmitted light. The difference between transmitted and captured light is an indication of relative $\mathrm{Chl}$ content per unit of leaf area (Demotes Mainard et al. 2008). Other indirect techniques, based on different spectral reflectance attributes, have been developed in recent years to determine the nitrogen status of plants. These indirect methods are usually implemented by sensors, such as the Dualex (Force- $A$, France) and Multiplex (Force-A, France) at leaf level, FieldSpec (ASD Inc., USA), CropScan L1 (CropScan Inc., USA), digital camera, Crop Circle (Holland Scientific Inc., USA), and Yara $N$ sensor (Yara, UK) at canopy level as well as Quick Bird (DigitalGlobe, USA), satellitemounted instrument, which have been also used as (remote) sensing techniques to estimate nitrogen in

Received 3 April 2016, accepted 20 September 2016, published as online-first 30 November 2016.

+Corresponding author; phone: +98 9123611832, fax:+98 2632808138, e-mail: omid@ut.ac.ir

Abbreviations: CCD - charged coupled device; Chl - chlorophyll content; CIE - international commission on illumination; DGCI dark green color index; GDR - green component divided by red component; GMR - green component minus red component; HSB hue, saturation, and brightness color space; ISO - sensor light sensitivity; LAI - leaf area index; LASAP - light aided spectral absorption photometry; LED - light emitting diode; NGI - normalized green index; MAE - mean absolute error; NGI -normalized green index; NRI - normalized red index; NRMSE - normalized root mean square error; NRI - normalized red index; RGB - red, green and blue color space; SAP - spectral absorption photometry; SPAD - soil plant analysis development; VI - vegetation index.

Acknowledgment: The financial support provided by the Research Department of University of Tehran, Iran, under grant No. 1305051.6.29 is duly acknowledged. 
different plants (Muñoz Huerta et al. 2013). Among these sensors, digital cameras are the cheapest. However, digital cameras have some often encountered problems for their use as sensors estimating nitrogen contents.

For example, differences in light conditions, such as shadow and sunny conditions, can affect results. In order to avoid it, solutions can be found in using a cover and adding an artificial light (Tewari et al. 2013). Another approach is based on exploiting combination of vegetation indices extracted from images in order to reduce the effect of variations in natural radiation under field conditions. Wang et al. (2013) used different indices including GMR (G minus R), G/R, NGI (normalized green index), NRI (normalized red index), and Hue indices. They mentioned that GMR and G/R indices show a higher correlation than other indices for estimation of biomass, Chl content, and leaf area index (LAI). The dark green color index (DGCI) was introduced based on the hue, saturation, and brightness (HSB) color space, and has been used to investigate the correlation of $\mathrm{Chl}$ and nitrogen content of plants (Karcher and Richardson 2003, Rorie et al. 2011a, Rorie et al. 2011b).

\section{Materials and methods}

Plant material and experimental site: The data were collected in maize plots at a Field Extension Education Laboratory, and a greenhouse located in Iowa State University, Ames, IA (USA) during the 2014 growing season. In order to simulate nitrogen deficiency in corn plants, different doses of nitrogen fertilization were applied in the field $\left(56,112,168\right.$, and $\left.224 \mathrm{~kg} \mathrm{ha}^{-1}\right)$ and inside a greenhouse. During data acquisition, field meteorological conditions including solar radiation, air temperature and relative humidity varied from 200 to $750 \mathrm{~W} \mathrm{~m}^{-2}, 20$ to $30^{\circ} \mathrm{C}$, and 65 to $95 \%$, respectively. For validation of the SPAD readings with $\mathrm{Chl}$ concentration, some experiments were carried out at the Agronomy Department of University of Tehran.

Data acquisition: Since our intention was to evaluate the capability of using smart phones as an instrument to estimate Chl content index (SPAD value), an Android smart phone was used to take images. The smartphone camera consisted of a 13 mega pixel charged-coupled device (CCD). In order to avoid the variability induced by different ambient conditions on data a new method of data acquisition by smartphone camera was presented, which we referred as spectral absorption photometry (SAP). For this method, leaves are held in front of the smartphone camera objective. In order to increase the accuracy of the SAP method, a part of data was acquired with background light source illumination, named, light-aided spectral absorption photometry (LASAP). For SAP, data were acquired under different light conditions. The settings of camera, including sensor light sensitivity (ISO) and
On the other hand, after a huge progress in both software and hardware of smart phones, their capabilities, especially in processing and imaging, have increased and new opportunities are created to take advantage of smart phones in many areas of research including agriculture (Moonrungsee et al. 2016, Pongnumkul et al. 2015). In recent study, a smart phone was used to determine total Chl content including Chl $a$ and $b$ and carotenoid (Car) content of soybean plants (Rigon et al. 2016). The extracted features from images have shown a high correlation with $\mathrm{Chl}$ and Car content.

Summarizing, the recent developments in using smart phones bring new opportunities to implementation and use of their capabilities, having different sensors and enabling different applications, and enabling measurements of various agricultural variables. In the present study, we evaluated the capability of smart phones to measure accurately the chlorophyll content in maize leaves. Thus, a new, cost-effective technique of imaging and spectral absorption photometry (SAP) was introduced.

exposure time, were set in auto mode and the camera selected them based on light conditions. In the LASAP mode, a light source containing 334-15/T1C1-LED was used to provide a constant illumination condition during imaging. The LEDs emitted white light according to CIE1931; the emitted-light chromaticity coordinates were $\mathrm{x}=0.30$ and $\mathrm{y}=0.29$ (Lights 2007). With the LASAP method, in order to gather the data, leaves were collected and images were captured from leaves on top of the light source with background illumination (Fig. 1). In this mode, unlike the SAP mode, all settings of the camera including ISO, exposure time, etc. were kept constant and during imaging, a white balance (color balance) was set for sunny conditions.

Standard imaging, which has been used in previous studies (de Souza et al. 2010) to estimate nitrogen deficiency and Chl content, entailed some issues, such as a time-consuming process due to analytical procedures for background segmentation and probable need for a setup or holder for the digital camera to keep a constant distance of the camera from the plants (de Souza et al. 2010).

For this study, 180 images were acquired using the SAP approach in the field of maize. About 90 images were also acquired through the LASAP approach from corn leaves. For both types of data acquisition, $L G$ E975 smart phone (LG Electronics, Korea) with a Kit Kat version of Android was the device which was used to acquire data. For light background illumination, a LED light source was used for providing extra light to acquire data. Fig. 2 shows some images based on the SAP and LASAP methods from corn leaves. 

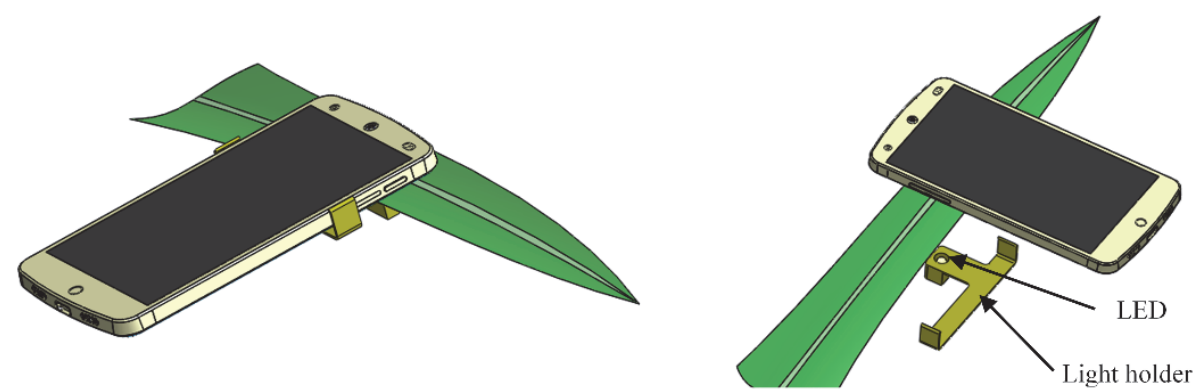

Fig. 1. The way of light background SAP by mobile phone in two views; without background light (left) and with light source (right).

SAP



LASAP

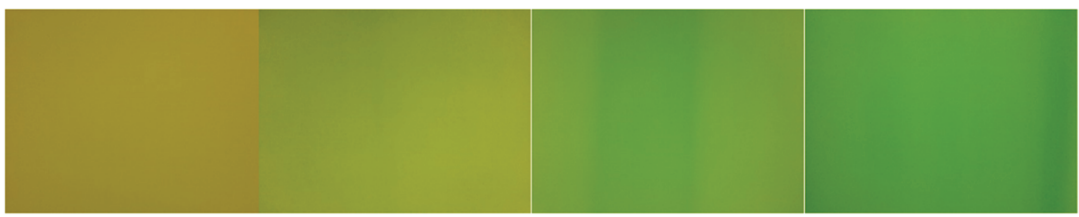

Fig. 2. Samples of spectral absorption photometry (SAP) and light-aided SAP (LASAP) from corn leaves under different levels of nitrogen fertilizing.

Determination of $\mathrm{Chl}$ content: To determine in vivo $\mathrm{Chl}$ content of corn leaves, a SPAD-502 (Minolta Co. Ltd., Osaka, Japan) was used. Chl-measuring instruments measure SPAD values, and these SPAD values have the following empirical (exponential) relation (Eq. 1) with Chl content in plants (Markwell et al. 1995):

$$
\text { Chl }=10^{\mathrm{M}^{\wedge} 0.265}
$$

where $\mathrm{Chl}$ is chlorophyll content in $\left[\mu \mathrm{mol} \mathrm{m} \mathrm{m}^{-2}\right]$ and $\mathrm{M}$ is the SPAD value measured by a Minolta SPAD 502.

In order to evaluate the Chl content given by Eq. (1), an experiment was carried out at the Agronomy Department of University of Tehran and the SPAD readings from 13 maize leaves were correlated to the extracted $\mathrm{Chl}$ concentrations. To measure Chl concentration from each leaf near the section that was read by a SPAD meter, a $1.6-\mathrm{cm}^{2}$ disc was submerged in $15 \mathrm{ml}$ of $80 \%$ acetone for $10 \mathrm{~min}$ and then the samples were placed in a centrifuge. A spectrophotometer (Cary 50, Varian, Australia) was used to determine the absorbance at two wavelengths (646.6 and $663.6 \mathrm{~nm})$, and then Chl concentration was calculated by method of Porra et al. (1974).

Vegetation indices used to determine Chl content: Estimation of the $\mathrm{Chl}$ content by images requires regularly the calculation of a number of indices from the images. This is usually more accurate than just using single channel spectral absorbance $(\mathrm{R}, \mathrm{G}, \mathrm{B})$. In this study, in addition to extracting R, G, and B components with Image Processing Toolbox of Matlab software (R2015 version 8.2), other principle components, such as hue, saturation, and brightness from HSB color space were calculated. Furthermore, some combined indices suggested by previous studies were considered, as well. These are GMR (difference between the green component and red component), GDR (green divided by red), VI (vegetation index, $(\mathrm{G}-\mathrm{R}) /(\mathrm{G}+\mathrm{R})$ ), and DGCI (dark green color index) (Kawashima and Nakatani 1998, Li et al. 2010, Tewari et al. 2013, Wang et al. 2013). For the calculation of these indices equations (2)-(8) were used.

$$
\text { Hue }=\left\{\begin{array}{cc}
60 \times\left(\frac{\mathrm{G}-\mathrm{B}}{\max (\mathrm{R}, \mathrm{G}, \mathrm{B})-\min (\mathrm{R}, \mathrm{G}, \mathrm{B})}\right), & \max (\mathrm{R}, \mathrm{G}, \mathrm{B})=R \\
60 \times\left(2+\frac{\mathrm{B}-\mathrm{R}}{\max (\mathrm{R}, \mathrm{G}, \mathrm{B})-\min (\mathrm{R}, \mathrm{G}, \mathrm{B})}\right), & \max (\mathrm{R}, \mathrm{G}, \mathrm{B})=G \\
60 \times\left(4+\frac{\mathrm{G}-\mathrm{B}}{\max (\mathrm{R}, \mathrm{G}, \mathrm{B})-\min (\mathrm{R}, \mathrm{G}, \mathrm{B})}\right), & \max (\mathrm{R}, \mathrm{G}, \mathrm{B})=B
\end{array}\right.
$$




$$
\begin{aligned}
& \text { Saturation }(S)=\left\{\begin{array}{cl}
\frac{\max (R, G, B)-\min (R, G, B)}{\max (R, G, B)}, & V=0 \\
\text { Brightness }\left(B^{\prime}\right)=\frac{\max (R, G, B)}{255} & V \neq 0
\end{array}\right. \\
& \text { GMR }=G-R \\
& \text { GDR }=\frac{G}{R} \\
& I=\frac{G-R}{G+R} \\
& \text { DGCI }=\left[\left(\frac{\text { Hue }}{60}-1\right)+(1-S)+\left(1-B^{\prime}\right)\right] / 3
\end{aligned}
$$

Since contact images were used, all R, G, and B in the above equations represented an average of pixel values of each image's component. Overall minimum and maximum values of mean and standard deviation of all ten indices were extracted for both types of contact images as shown in the following text table.

The minimum and maximum value of averages and standard deviations (SD) of extracted indices, using spectral absorption photometry (SAP) and light-aided SAP (LASAP) approaches.

\begin{tabular}{lllllllll}
\hline & $\begin{array}{l}\text { SAP } \\
\text { Mean } \\
\text { Min }\end{array}$ & Max & $\begin{array}{l}\text { SD } \\
\text { Min }\end{array}$ & Max & $\begin{array}{l}\text { LASAP } \\
\text { Min }\end{array}$ & Max & Min & Max \\
\hline R & 0.17 & 148.87 & 0.49 & 20.60 & 81.02 & 163.04 & 3.90 & 20.41 \\
G & 144.56 & 190.75 & 2.19 & 17.49 & 142.16 & 163.90 & 3.61 & 12.93 \\
B & 0.00 & 20.07 & 0.00 & 17.24 & 0.23 & 1.63 & 0.44 & 1.09 \\
Hue & 10.44 & 20.81 & 0.36 & 1.11 & 8.85 & 15.06 & 0.13 & 1.03 \\
S & 0.88 & 1.00 & 0.00 & 0.10 & 0.99 & 1.00 & 0.00 & 0.01 \\
B' & 0.57 & 0.75 & 0.01 & 0.07 & 0.58 & 0.64 & 0.01 & 0.05 \\
GMR & 6.9 & 168.15 & 1.15 & 19.36 & -18.58 & 81.6 & 2.07 & 15.53 \\
GDR & 1.05 & 988.29 & 0.84 & 30.54 & 0.89 & 2.01 & 0.01 & 0.31 \\
VI & 0.02 & 1.00 & 0.01 & 0.15 & -0.06 & 0.33 & 0.01 & 0.08 \\
DGCR & -0.17 & -0.08 & 0.00 & 0.04 & -0.163 & -0.12 & 0.01 & 0.02 \\
\hline
\end{tabular}

Evaluating SAP and LASAP methods: To evaluate both types of presented data-acquisition (SAP and LASAP) techniques, the mean of all indices including the RGB color space and the HSB color space and other indices were correlated with $\mathrm{Chl}$ content obtained with a SPAD meter according to Eq. 1. Regression parameters were obtained for both types of imaging and coefficient of

\section{Results and discussion}

Validation of $\mathrm{Chl}$ content determination: The Chl concentration of 13 corn leaves was determined by the experimental method described above and SPAD meter readings were correlated to it. As mentioned in literature (Markwell et al. 1995), values achieved by the Eq. 1 were well correlated to experimental results. Fig. 3 shows the scatter plot between the actual and estimated $\mathrm{Chl}$ content and their correlation. The $R^{2}$ for Eq. 1 was calculated as 0.9 .

\section{Correlation of indices determined by SAP with Chl 606}

determination ( $r$-square), normalized root mean square error (NRMSE; 0 to 1 , with optimum equaling 0 ), and the mean absolute error (MAE; 0 to $+\infty$, with optimum equaling 0) (Willmott et al. 1985), were computed within Matlab environment (Mathworks Inc.) as performance factors for each index.

content: In order to evaluate the ability of SAP method for Chl content estimation in corn plants, the coefficient of determination for each index was calculated (Table 1). Correlation of each index with the Chl content for the SAP method is shown in Fig. 3. According to $R^{2}$ values in Table 1, the Hue index correlated with the Chl content slightly better than the other indices, but for most of indices the SAP method estimated the Chl content inaccurately. In contrast to previous studies, the green parameter was not an appropriate index to estimate the Chl content in the SAP 


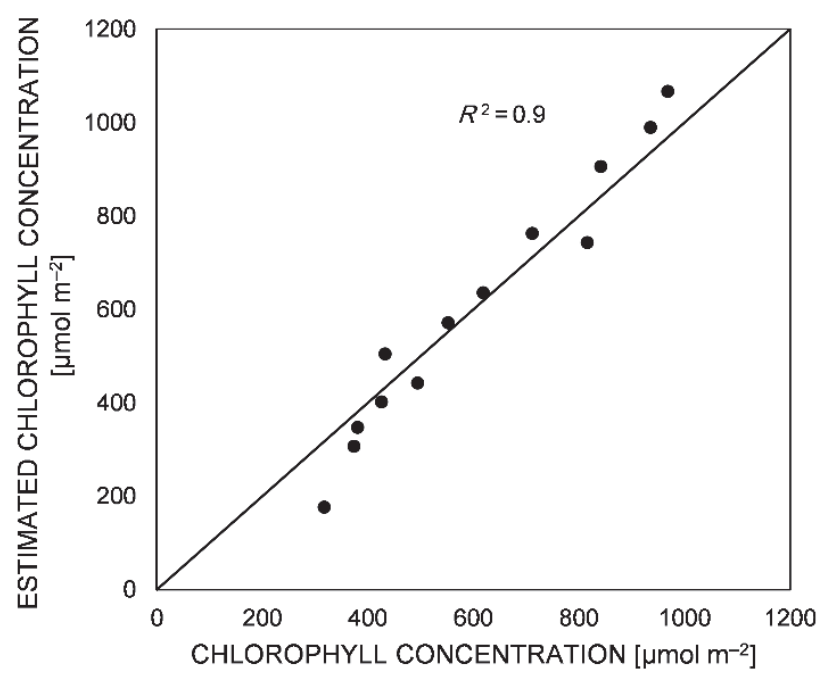

Fig. 3. Correlation of chlorophyll concentrations and SPAD meter-estimated chlorophyll content, calculated by Eq. 1 . method because of its low $r$-square value (Rorie et al. 2011b). Among red, green, and blue components of RGB color space indices of SAP, R parameter $\left(R^{2}=0.47\right)$ fitted to the Chl content much better in contrast with the $\mathrm{G}$ $\left(R^{2}=0.08\right)$, and $\mathrm{B}\left(R^{2}=0.09\right)$ components, respectively. Likewise, Hue, GMR, and VI with $R^{2}$ values of $0.5,0.48$, and 0.42 , respectively, gave a better performance for the Chl content estimation than other indices (Fig. 4). Finally, the DGCI index (Table 1), which was mentioned as a good index for imaging in order to estimate nitrogen status in regular images (Karcher and Richardson 2003), did not have a high enough correlation for the SAP method described in this article.

The data acquisition in SAP mode, which was performed under natural field conditions with (a regular) smart phone without any supplementary illumination, led to acceptable and accurate results. This is in contrast to some other studies that reached almost the same results while they were performed with a data acquisition under

Table 1. Regression parameters including slope, intercept and coefficient of determination $\left(R^{2}\right)$, MAE (mean absolute error), and NRMSE (normalized room mean square error) of different indices correlated to chlorophyll content of both SAP (spectral absorption photometry) and LASAP (light-aided spectral absorption photometry). VI in LASAP images correlated more to chlorophyll content than other indices.

\begin{tabular}{|c|c|c|c|c|c|c|c|c|c|c|}
\hline \multirow[t]{3}{*}{ Indice } & \multicolumn{5}{|c|}{ Regression parameter } & \multicolumn{3}{|c|}{ MAE } & \multicolumn{2}{|c|}{ NRMSE [\%] } \\
\hline & Slope & & Intercept & & $R^{2}$ & & & & & \\
\hline & SAP & LASAP & SAP & LASAP & SAP & LASAP & SAP & LASAP & SAP & LASAP \\
\hline Red & -5.48 & -11.41 & $1,065.78$ & $1,956.31$ & 0.47 & 0.94 & 131.00 & 45.34 & 16.44 & 5.61 \\
\hline Green & 11.21 & 21.48 & $-1,194.29$ & $-2,804.54$ & 0.08 & 0.21 & 171.35 & 168.85 & 21.63 & 21.09 \\
\hline Blue & 19.70 & 18.24 & 567.60 & 541.98 & 0.09 & 0.00 & 169.92 & 189.89 & 21.5 & 23.68 \\
\hline Hue & 84.75 & 164.27 & -652.44 & $-1,439.74$ & 0.50 & 0.96 & 126.17 & 41.06 & 15.97 & 4.99 \\
\hline Saturation & $-2,856.81$ & $-2,115.98$ & $3,430.08$ & $2,661.22$ & 0.07 & 0.00 & 171.34 & 189.67 & 21.77 & 23.68 \\
\hline Brightness & $2,857.81$ & 2406.03 & $-1,194.20$ & -928.55 & 0.08 & 0.03 & 171.36 & 182.25 & 21.63 & 23.33 \\
\hline GMR & 5.20 & 10.35 & 205.54 & 207.35 & 0.48 & 0.96 & 127.19 & 41.32 & 16.22 & 4.99 \\
\hline GDR & 0.33 & 942.57 & 605.39 & -681.28 & 0.02 & 0.96 & 179.54 & 35.43 & 22.37 & 4.64 \\
\hline VI & 859.24 & 2618.54 & 321.33 & 225.87 & 0.42 & 0.97 & 138.26 & 32.41 & 17.23 & 4.11 \\
\hline DGCI & $7,988.74$ & $2,0151.32$ & $1,595.42$ & $3,306.37$ & 0.25 & 0.57 & 154.38 & 119.42 & 19.55 & 15.52 \\
\hline
\end{tabular}

constant laboratory conditions (Gupta et al. 2013, Liu et al. 2010).

Correlation of LASAP method indices with Chl content: The accuracy of the LASAP approach in estimating $\mathrm{Chl}$ content was evaluated by correlating the extracted indices with measured leaf $\mathrm{Chl}$ contents. High performance of the LASAP method to estimate the Chl content was obvious for most of the indices (Fig. 4), and also regression parameters for indices of LASAP method confirmed this superior performance (Table 1). For the LASAP (similarly as SAP) method, the R component from the RGB color space and the Hue component from HSB color space followed by GMR, GDR, and VI from combined indices were better correlated than the other ones. The best index in this study was the VI index with $R^{2}$ $=0.97$ (Table 1). For LASAP data, DGCI did not elicit a high enough correlation with the $\mathrm{Chl}$ content. This might indicate that in both methods, DGCI did not represent the Chl content. High values of $R^{2}$ showed the methods described here to be a plausible alternative to estimate the Chl content with a high accuracy. This method achieved a relatively high precision compared to other methods that have been applied in previous studies (Wang et al. 2014, Yadav et al. 2010). In comparison with these studies, the procedure used in our study was easier. For instance, in Liu et al. (2010), research spectral indices at different wavelengths were used as input features to neural networks to estimate Chl content. $R^{2}$ for the neural network was 0.90 , while their best index reached the $R^{2}$ value of 0.73 . The presented LASAP method based on a regular smart phone and simple back lighting (Fig. 1) reached more accurate results (Fig. 5). In other words, using this method in addition to giving accurate results, eliminated the 

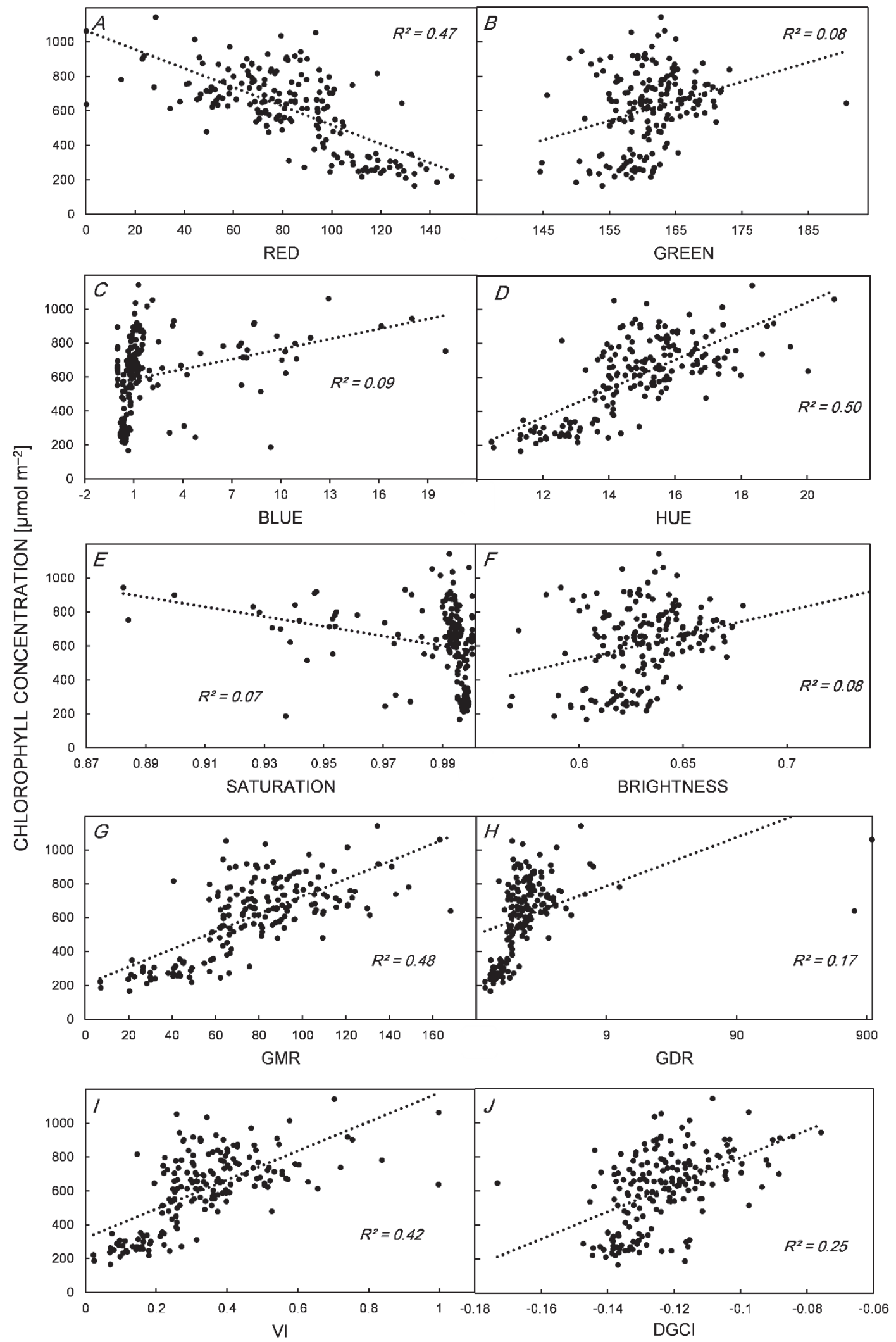

Fig. 4. Correlation of red $(A)$, green $(B)$, blue $(C)$, hue $(D)$, saturation $(E)$, brightness $(F)$, green component minus red component (GMR, $G)$, green component divided by red component (GDR, $H$ ), vegetation index (VI, $I$ ), and dark green color index (DGCI, $J$ ) indices extracted from SAP data with chlorophyll content.

608 

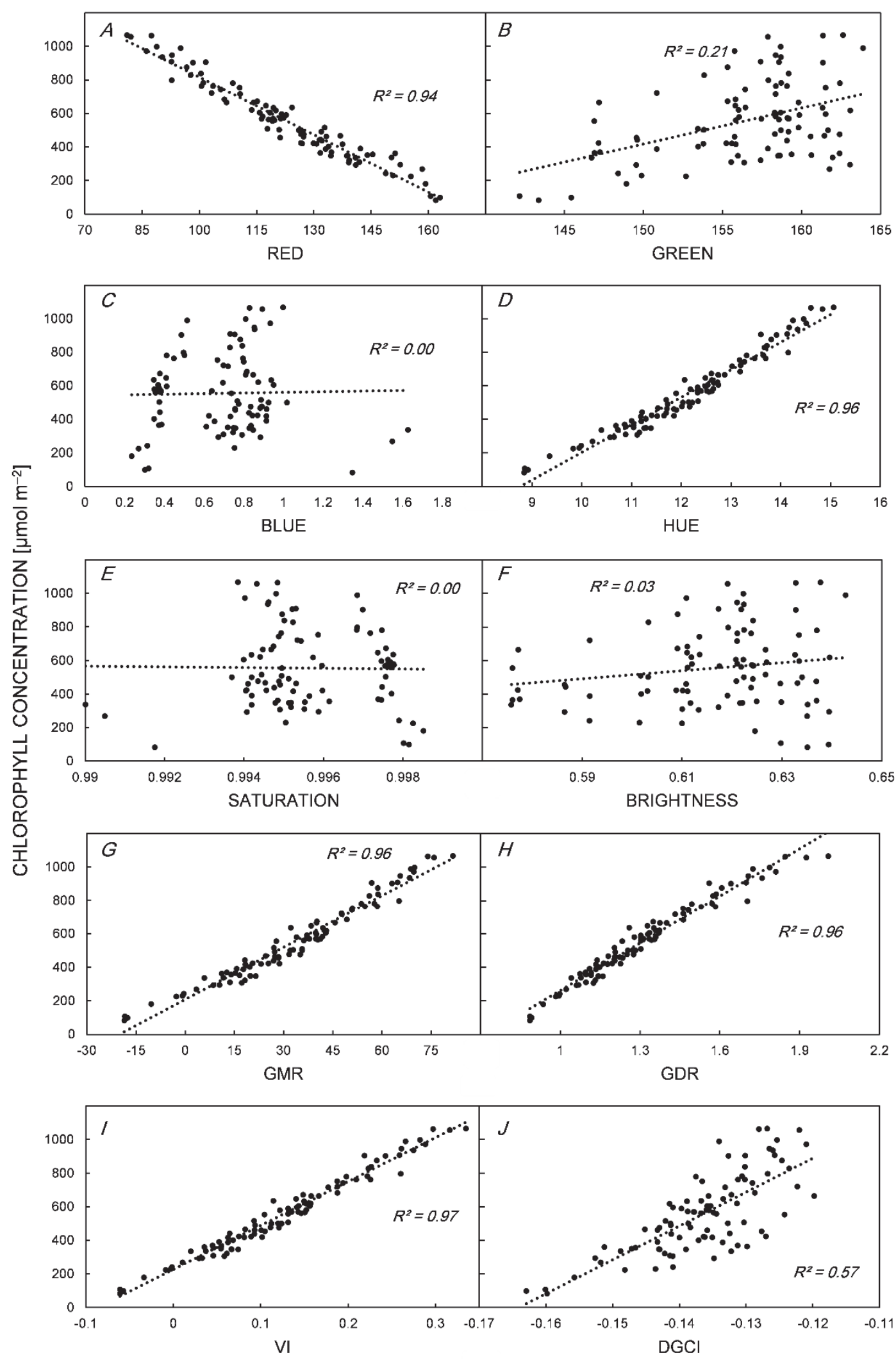

Fig. 5. Correlation of red $(A)$, green $(B)$, blue $(C)$, hue $(D)$, saturation $(E)$, brightness $(F)$, green component minus red component (GMR, $G$ ), green component divided by red component (GDR, $H$ ), vegetation index (VI, $I$ ), and dark green color index (DGCI, $J$ ) indices extracted from LASAP data with chlorophyll content. 
requirement for background segmentation and the need for a fixed laboratory conditions and special setups.

Conclusions: In order to increase capability of digital cameras to estimate the chlorophyll content, a new way of data acquisition was adapted for acquiring data through smartphone camera from corn leaves. Spectral absorption photometry, light-aided and without background light (LASAP and SAP) images from corn leaves under various nitrogen fertilization were acquired with a smart phone. In LASAP mode, the obtained vegetation index showed the highest correlation coefficient with respect to other indices with a $R^{2}$ value reaching 0.97 . These results indicated that by implementing this method as an smartphone application

\section{References}

de Souza E.G., Scharf P.C., Sudduth K.A.: Sun position and cloud effects on reflectance and vegetation indices of corn. Agron J. 102: 734, 2010.

Demotes-Mainard S., Boumaza R., Meyer S., Cerovic Z.G.: Indicators of nitrogen status for ornamental woody plants based on optical measurements of leaf epidermal polyphenol and chlorophyll contents. - Sci Hortic.-Amsterdam 115: 377-385, 2008.

Dutta Gupta S., Ibaraki Y., Pattanayak A.K.: Development of a digital image analysis method for real-time estimation of chlorophyll content in micropropagated potato plants. - Plant Biotechnol. Rep. 7: 91-97, 2013.

Evans J.R.: Photosynthesis and nitrogen relationships in leaves of C3 plants - Oecologia 78: 9-19, 1989.

Karcher D.E., Richardson M.D.: Quantifying turfgrass color using digital image analysis. - Crop Sci. 43: 943-951, 2003.

Kawashima S., Nakatani M.: An algorithm for estimating chlorophyll content in leaves using a video camera. - Ann BotLondon. 81: 49-54, 1998.

Li Y., Chen D., Walker C.N., Angus J.F.: Estimating the nitrogen status of crops using a digital camera. - Field Crop. Res. 118: 221-227, 2010.

Lights L.A.: 334-15/T1c1-4wya led Datasheet. Pp. 1-10. Everlight Electronics Co., Ltd., New Taipei 2007.

Liu M., Liu X., Li M. et al.: Neural-network model for estimating leaf chlorophyll concentration in rice under stress from heavy metals using four spectral indices. - Biosyst. Eng. 106: 223233, 2010.

Markwell J., Osterman J., Mitchell J.: Calibration of the Minolta SPAD-502 leaf chlorophyll meter. - Photosynth. Res. 46: 467472, 1995.

Miao Y., Mulla D.J., Randall G.W. et al.: Combining chlorophyll meter readings and high spatial resolution remote sensing images for in-season site-specific nitrogen management of corn. - Precis. Agric. 10: 45-62, 2009.

Moonrungsee N., Pencharee S., Peamaroon N.: Determination of iron in zeolite catalysts by a smartphone camera-based colorimetric analyzer. - Instrum. Sci. Technol. 44: 401-409, 2016.

Muñoz-Huerta R.F., Guevara-Gonzalez R.G., Contreras-Medina L.M. et al:: A review of methods for sensing the nitrogen status in plants: advantages, disadvantages and recent advances. - and using a small attached lighting device, a smart phone was shown to be an accurate and practical alternative to the higher cost SPAD meters. In addition to the low cost, due to the presence of different sensors and powerful processors in last generation of smart phones, other applications can be envisaged in a simultaneous mode, such as the acquisition of continuous chlorophyll content data sets, combined with the logging of GPS data or other capabilities that can be found on smart phones. Furthermore, in comparison with other methods based on the use of digital cameras, using smart phones and spectral absorption photometry eliminates the need for a computer or desktop, which helps to make the approach more accurate, simple, and easy to use anywhere at any time.

Sensors 13: 10823-10843, 2013.

Pongnumkul S., Chaovalit P., Surasvadi N.: Applications of smartphone-based sensors in agriculture: a systematic review of research. - J. Sensor. 15: 18-26, 2015.

Porra R.J., Grimme L.H.: A new procedure for the determination of chlorophylls and band its application to normal and regreening chlorella. - Anal. Biochem. 57: 255-267, 1974.

Rigon J.P.G., Capuani S., Fernandes D.M., Guimarães T. M.: A novel method for the estimation of soybean chlorophyll content using a smartphone and image analysis. - Photosynthetica 54: 559-566, 2016.

Rorie R.L., Purcell L.C., Karcher D.E., King C.A.: The assessment of leaf nitrogen in corn from digital images. - Crop Sci. 51: 2174-2180, 2011a.

Rorie R.L., Purcell L.C., Mozaffari M. et al.: Association of "greenness" in corn with yield and leaf nitrogen concentration. - Agron. J. 103: 529-535, 2011 b.

Tewari V.K., Aruda A.K., Kumar S.P. et al.: Estimation of plant nitrogen content using digital image processing. - Agric. Eng. Int. CIGR J. 15: 78-86, 2013.

Turner R.E., Rabalais N.N.: Changes in Mississippi river water quality this century. - BioScience 41: 140-147, 1991.

Vesali F., Omid M., Kaleita A., Mobli H.: Development of an Android app to estimate chlorophyll content of corn leaves based on contact imaging. - Comput. Electron. Agr. 116: 211220, 2015.

Wang Y., Wang D., Shi P., Omasa K.: Estimating rice chlorophyll content and leaf nitrogen concentration with a digital still color camera under natural light. - Plant Methods 10: 1-11, 2014.

Wang Y., Wang D., Zhang G., Wang J.: Estimating nitrogen status of rice using the image segmentation of G-R thresholding method. - Field Crop. Res. 149: 33-39, 2013.

Willmott C.J., Ackleson S.G., Davis R.E. et al.: Statistics for the evaluation and comparison of models. - J. Geophys. Res.Oceans 90: 8995-9005, 1985.

Yadav S., Ibaraki Y., Dutta Gupta S.: Estimation of the chlorophyll content of micropropagated potato plants using RGB based image analysis. - Plant Cell Tiss. Organ Cult. 100: 183$188,2010$. 